\title{
Educación Superior Dominicana: tendencias y desafíos ${ }^{\mathrm{A}}$
}

\author{
Dominican Higher Education: trends and challenges
}

\author{
Fernando I. Ferrán ${ }^{B}$
}

Recibido: 10/6/2019 • Aprobado: 31/7/2019

Cómo citar: Ferrán, F. I. (2019). Educación Superior Dominicana: tendencias y desafíos. Ciencia y Educación, 3(2), 7-17. Doi: https://doi.org/ 10.22206/cyed.2019.v3i2.pp7-17

Resumen

Este ensayo plantea una caracterización del sistema contemporáneo de educación superior en la República Dominicana, a partir de sus principales indicadores estadísticos de desempeño durante los últimos decenios y de la puesta en evidencia de sus limitantes en un contexto global. El propósito es discernir, primero, su concepción fundacional o modelo originario; segundo, establecer sus puntos operativos más críticos en un ámbito supranacional; finalmente, redefinir su naturaleza y quehacer, tanto en función de su razón de ser, en términos conceptuales y sociales, como de las tendencias prevalecientes y de los principales desafíos prácticos que enfrenta en la actualidad.

Palabras clave: desempeño; enseñanza profesional; modelo educacional; enseñanza; aprendizaje; mercado de trabajo.

\begin{abstract}
This essay characterizes the contemporary system of higher education in the Dominican Republic based on its main statistical indicators of performance during the last decades, as well as the demonstration of its limitations in a global context, in order to discern, first, its foundational conception or original model; second, to establish its most critical operational points in a supranational environment; and finally, third, to redefine its nature and work, both in terms of its rationale in conceptual and social terms, as well as the prevailing trends and the main practical challenges it faces today.
\end{abstract}

Keywords: performance; professional education; educational model; teaching; learning; Work market

A. Elaborado a partir de la ponencia presentada en el Seminario Internacional de Educación Superior. La Investigación y la Evaluación Educativa en América Latina y el Caribe. Avances, tendencias y desafíos en el Siglo XXI, bajo la dirección de la Universidad Católica Tecnológica del Cibao (UCATECI) y del Instituto Superior de Estudios Educativos Pedro Poveda - ISESP, el 4 de abril de 2019.

B. Antropólogo social y filósofo, investigador y profesor del Centro de Estudios Económicos y Sociales Padre José Luis Alemán de la Pontificia Universidad Católica Madre y Maestra (PUCMM). Correo-e: fernandoferran@pucmm.edu.do 
La educación vale. Las ideas impactan. Muy particularmente, en un contexto contemporáneo de civilización en el que la competencia es ley y la toma de decisiones edificadas se presenta como una necesidad, en donde la revalorización de la existencia humana un imperativo impostergable.

He ahí el motivo por el que me intereso en el tema de la educación superior dominicana (ESD) y su sistema operativo (SESD).

En ese contexto, antes de iniciar la exposición, pongo sobre el tapete los dos axiomas a partir de los que abordaré la cuestión que me preocupa, esto es, la finalidad de la ESD a partir de su realidad, de su tendencia y de sus desafíos:

1. El valor de ningún centro de ESD está por encima de la calidad de sus profesores.

Por vía de consecuencia, la calidad de la ESD en general, así como el valor de cada una de sus instituciones en particular, es directamente proporcional a la excelencia y perfección $-\mathrm{o}$ irrelevancia e imperfección- de sus docentes.

2. El SESD es suigeneris, por no decir que inexistente en términos académicos, pues se estructura a partir de carencias, dada la ausencia de una relación continua y de una promoción recíproca entre profesores e investigadores, de un lado; del otro, estudiantes y sociedad en general.

\section{El Sistema de Educación Superior Dominicano (SESD)}

Una buena y sucinta síntesis objetiva del desempeño del SESD aparece en el estudio Educación superior dominicana: expansión desarrollo y perspectivas futuras, realizado por el Observatorio de Políticas Sociales y Desarrollo de la Vicepresidencia de la República (2019). A continuación, algunos de los datos y hallazgos más relevantes de los ahí expuestos.

a. Modelo en transición. El SESD está en transición: del elitismo intelectual a un bien público masificado. Ese proceso depende del paradigma posindustrial y del poscapitalista de acuerdo al cual el uso intensivo de la fuerza habría de ser reemplazado por el uso intensivo de conocimiento (Bell, 1976; Drucker, 1993). En ese contexto, la alternativa hipotética es la siguiente: rebasar la era industrial o, como advierte Toffler (1984), aunque en otro contexto, seguir siendo una academia únicamente profesionalizante donde solo salen mal o escasamente preparados estudiantes para la era industrial. (Observatorio, 2019, p. 4)

b. Matrícula. El incremento de la matrícula en los centros de educación superior en el país es notable, al igual que el de su cobertura. Entre los años 1993 y 2002 el país ha pasado de una matrícula de 108,335 a 286,134 estudiantes. Y de principios de la década de 1990 al año 2017 , la tasa de cobertura se incrementó de $10 \%$ a $60.58 \%$, bien por encima del promedio latinoamericanos (50\%) y del mundo (38\%). (Observatorio, 2019, p. 6)

c. Recursos financieros. Ese vertiginoso y notable aumento de los indicadores formales del sistema viene sustentado por un gasto/inversión promedio de $0.4 \%$ anual del PIB una vez pasa de RD\$19,528 en 2013, a RD\$24,314 en 2017. A una tasa de RD\$50/US\$1, la inversión por estudiante en el país es de poco menos de US\$ 500, en contraste con el promedio de la región OCDE de US\$16,400, y en particular Chile (US\$ 8,500), México (US\$ 9,500) y Corea del Sur (US\$ 9,700). (Observatorio, 2019, pp. 6-7)

d. Perfil de ingreso. En términos del perfil de ingreso, una limitante fundamental del SESD está en "el gran número de estudiantes con escasa preparación académica para el trabajo que conlleva la educación superior (...) por haber recibido una educación primaria y secundaria de baja calidad" (Observatorio, 2019, p. 5). Con razón de ahora en adelante habrá que darle seguimiento y trazar la tendencia a partir de las pruebas PISA, en las que la República Dominicana ocupa el último puesto de los 70 países evaluados en matemáticas, ciencias y lectura en el año escolar 2015-2016. ${ }^{\mathrm{A}}$

A. El promedio alcanzado por el país en 2015-2016 fue de 339.3 puntos. (Ver, http://factsmaps.com/pisa-worldwide-ranking-average-score-of-math-science-reading/). Es de interés en este punto cerrar el círculo. Quienes forman a esos 
e. Perfil del egresado. Y en términos del perfil del egresado, resalta el bajo nivel del índice de capital humano de la República Dominicana, igual a $0.49 \%$. Ese bajo índice es tan preocupante como su significado: "En promedio, un niño(a) dominicano(a) que nace hoy logrará solo el $49 \%$ de su potencial de productividad" (Observatorio, 2019, p. 8). Obviamente, el índice de capital humano ensombrece el presente y oscurece más el porvenir. ${ }^{\mathrm{B}}$

f. Profesores. Testigos de excepción del proceso de generación-enseñanza-aprendizaje, se reportan 20,291 profesores universitarios. De ese total del claustro profesoral, el $10 \%$ está contratado a tiempo completo, un $3.94 \%$ posee doctorado y solo un $3.1 \%$ (618) de ellos desarrollan actividades de investigación. (Observatorio, 2019, p. 9)

g. Posicionamiento internacional. En ese horizonte de cosas, ninguna institución de educación superior dominicana aparece en las posiciones cimeras de los rankings internacionales. Peor aún; en el índice internacional de universidades contemporáneas aparecen menos de cincuenta instituciones de educación superior iberoamericanas — ninguna de ellas dominicana — entre las 1000 mejores del mundo de acuerdo a tres de las principales clasificadoras de mayor relevancia en el ambiente académico internacional (Observatorio, 2019, Tabla 1). ${ }^{\mathrm{C}}$

estudiantes preuniversitarios son maestros preparados en aulas de los centros de educación superior. Por eso el instructivo artículo de Celeste Abreu (2018) ponderando las más recientes políticas docentes en el país. Al respecto, retengo de la autora esta conclusión: "No obstante, sin desconocer los avances alcanzados en planes, programas y estrategias que plantean como prioridad el tema docente, hay una distancia entre esas formulaciones y su nivel de concreción. Diríase que el tiempo político no coincide con el tiempo técnico-pedagógico para hacer realidad las aspiraciones expresadas en los referidos marcos de planteamiento. El caso de la propuesta del Sistema de Carrera Docente es sólo una muestra de esa situación" (Abreu, 2018: pp. 25-26).

B. Para algunos, como Andrés Oppenheimer, la solución de tal limitante pudiera ser "producir más ingenieros y científicos, y tal vez menos filósofos" (2018, p. 6). Claro está, todo va a depender de qué significa para él "producir" profesionales. Independientemente de la respuesta y de su concepción kanteana de la educación superior, más relevante me parece ser el posicionamiento del sistema educativo contemporáneo con una sociedad humana regenteada por la inteligencia artificial. A este propósito, ver, por ejemplo, los argumentos y contraargumentos expuestos durante el diálogo que a ese propósito sostuvieron Chalmers y Dennet, (2019).

C. Las tres agencias son: ARWU, Times Higher Education y Quacquarelli Symonds. (Ver Observatorio 2019: Tabla 1).
Al certero diagnóstico, hasta aquí presentado, conviene ańadir algunas variables inseparables de cualquier sistema de enseñanza. Por ejemplo:

h. Evaluación. Valdría la pena indagar si el sistema de pruebas y de exámenes regulares que evalúan el aprendizaje en la ESD logra algo más que cercenar, en el altar unidimensional de la mera objetividad, la imaginación, la creatividad y la capacidad singular de cada estudiante para resolver problemas (Giroux, 2008). ${ }^{\mathrm{D}}$

i. Investigación. El Ministerio de Educación Superior, Ciencia y Tecnología realiza una labor encomiable incentivando los trabajos de pesquisa en centros especializados y de educación superior. Durante los últimos catorce años (2005-2018), ha asignado a labores de investigación en instituciones de educación superior (IES) — privadas y estatales - un promedio anual de RD\$211.5 millones, equivalente al $1.6 \%$ de su presupuesto ministerial (Corcino, 2019, pp. 16-17).

j. Modelo institucional. En más de un sentido, las “infecciones sistémicas" que Gerald Murray (2005, pp. 363-364) descubre en los planteles escolares del país, aparecen también a nivel superior. No todo es —aunque tambiénescasez de recursos financieros, a menos que se quiera pasar por alto la centralización en la toma de decisiones en el sistema como tal y en cada institución en particular.

k. Mercado laboral. Durante el año 2016, EDUCA (2019) señala que el $21.5 \%$ de los jóvenes dominicanos entrevistados con edades comprendidas entre 15 y 29 años permaneció desempleado. Así las cosas, ese segmento poblacional engrosa la generación de los "Ni-Ni" —ni trabajan ni estudian-y de los "Sin-Sin", en referencia a las personas sin las competencias requeridas por el mercado de trabajo y sin oportunidades para acceder a una vida más

D. De ser así, el modo de evaluación estaría propiciando sujetos disciplinados, gregarios y predecible y, al mismo tiempo, desviando la atención del problema de fondo de la educación; a saber, "no fomentar el pensamiento crítico para crear ciudadanos 'conformistas' que no reclamen nada" ni a sus superiores en el sector público ni en el privado (ver la obra de Giroux 2003, 2005, 2008; y la reciente entrevista al citado académico canadiense en Menárguez, 2019). 
digna y próspera. Esa doble condición afecta en el país más a la población femenina (27\%) que a la masculina $12 \%)$.

En ese contexto, la certera conclusión del Observatorio de Políticas Sociales y Desarrollo de la Vicepresidencia de la República Dominicana queda aún mejor avalada:

1. Conclusión. "Sea porque las condiciones de trabajo justifican el pluriempleo, o sea por el bajo presupuesto para la investigación, o ambas, el perfil de la educación superior dominicana es predominantemente un sistema de otorgar credenciales, con muy pocas repercusiones en la producción de conocimiento" (Observatorio, 2019, p. 9).

Y, por consiguiente, si alguna tendencia salta a la vista en el modelo educativo vigente en el país es que el SESD, con sus 51 centros y más de 450 mil estudiantes, continúa a la baja en términos de eficiencia y de calidad. Ese declive queda al descubierto en y por el carácter sui generis de una estructura carenciada en la que no hay profesores ni estudiantes, tampoco soporte financiero ni publicaciones ni investigaciones y menos aún valoración y empleo de estas.

A todas luces, todo eso implica la perpetuación de un SESD de cuestionable valor en función del actual y sobre todo del venidero mundo cultural y su consecuente mercado laboral.

\section{Lo agujeros negros de la Educación Superior Dominicana, la ESD}

Los principales agujeros negros ${ }^{\mathrm{F}}$ de los que surgen los actuales límites del SESD y que hay que superar de una vez por todas son, al menos, los siguientes:

a. Realidad. Se desconoce cómo y por qué medios la ESD —otrora esperanza de mejoría y bienestar individual y social- contribuye a que la sociedad dominicana siga expulsando de su seno un número significativo de lo mejor de

\footnotetext{
E. El referido estudio de EDUCA (2019) consigna que el $41.6 \%$ de los jóvenes dominicanos con edades comprendidas entre 15 y 29 ańos no completa la secundaria y que del $58.4 \%$ de los que sí la finalizan, un cuarto de ellos (24\%) no se matriculan en el nivel superior. Ese comportamiento es atribuido por el estudio de referencia a la alta tasa de abandono escolar: el $37.7 \%$ de la población entrevistada dejó de asistir a la escuela o a la universidad.

F. A propósito del significado atribuido a dicho término en este contexto, consúltese Harari, 2018, p. 243 y ss.
}

su juventud y lo condicione a buscar sueños y realizar legítimas aspiraciones y derechos fuera del país. Esto acontece ante la mirada - ¿impotente o indiferente? - de todos, no obstante que un significativo $63 \%$ de la población entre 18 y 25 años de edad aspira a irse de su país (Latinobarómetro, 2018). ${ }^{\mathrm{G}}$

b. Concepción. No se reconoce que la ESD está mal concebida, mal fundada y al entender de otros, ${ }^{\mathrm{H}}$ mal evaluada.

b.1. Mal concebida. Dicha educación está "varada" por lo menos desde hace cinco o seis décadas en un modus operandi mercantil cuyo contexto ideológico y cultural es incapaz de reconocer que las personas - particularmente las más sobresalientesnunca han sido copias o versiones de alguien más. Y por eso mismo en el país - a no ser que se trate de algún caso de excepción que confirma la regla - nadie es promovido en las aulas de alguna de las instituciones de educación a nuevos sitiales de innovación en la era global de conocimientos, innovaciones y transformaciones revolucionarias. Sencillamente, la ESD deambula ignorando la modernidad y esquivando la globalización. ${ }^{\mathrm{I}}$

b.2. Mal fundada. Su proceso de enseñanza-aprendizaje es contradictorio. Habla de la confianza que deposita en el individuo y en su capacidad de aprendizaje del yo cartesiano, pero en la práctica cada sujeto queda relegado por toda una estructura institucional que privilegia en sus programas educativos el manejo de cohortes y promociones y es incapaz de descubrir la vocación profesional (beruf) singular de cada individuo en particular que es, según Weber (2012), la sal que aporta el espíritu capitalista al cuerpo social y económico.

G. Una aproximación encaminada a esclarecer la situación de frustración que resienten los nuevos profesionales en el país aparece en Tavares, 2019.

H. A modo de ejemplo, Cubero et al., 2014.

I. A propósito de esta y lo ajena que permanecen ciertos sistemas de formación superior, como el dominicano, de la nueva era que afecta, ver una visión de conjunto en García, 2016. 
b.3. Mal evaluada. La educación superior en la región iberoamericana en general, y en concreto en el país, es sacrificada en el altar de la objetividad cuantitativa y desconoce los intríngulis, no solo de una investigación mixta de métodos objetivos y cualitativos, sino de una evaluación igualmente cualitativa en función del proceso de asimilación subjetiva que resulta de la enseńanza-aprendizaje. Su unidimensionalidad se oculta en el tecnicismo y en las mediciones de volúmenes cuantitativos. Si bien estos son imprescindibles, — no se dude de eso— no significan una evaluación integral a través de actividades que potencien habilidades como la interacción grupal y que valoren no solo las habilidades cognoscitivas, sino también aspectos socio-afectivos como, por ejemplo, la actitud, la motivación, la colaboración y el gradual desarrollo vocacional de cada uno.

c. Desenfoque. Es improbable que la población beneficiaria de la ESD mejore por el mero hecho de que se le quiera proporcionar más y mejor información. El sujeto humano no necesita más datos e información, para eso está "Mr. Google" a distancia de un clic para procurarle más datos e información que la que puede proporcionarle cualquier centro de educación formal.

d. Desorientación. El énfasis puesto en la educación en competencias ha propiciado el modelo metodológico europeo de la Universidad empresa para responder a las nuevas necesidades del mercado laboral globalizado. Ese mercado instrumentaliza, de manera utilitaria, ciertas especialidades, pero prescinde por completo de la comprensión de las mismas y de cuanto signifique lo que autores como Bertrand Russel, Paulo Freire y otros, denominaban el ensanchamiento de la mente, el análisis imparcial del mundo o la socialización de sueños, utopías y valores compartidos. Debido a tal sesgo, la existencia humana reducida a expresión laboral y la formación del sujeto humano a la obtención de competencias laborales. Eso es necesario, mas insuficiente si obnubila o prescinde de "una previa reflexión crítica sobre qué significan, cuál es su origen y de dónde proceden (las competencias), cuál es su contexto ideológico y, sobre todo, las incompatibilidades y contradicciones entre el modelo de saberes y destrezas propios de ellas y el conocimiento científico, teórico y crítico" (Vargas, 2019, párr. 4).

e. Carencias. Por vía de consecuencia, lo que el sujeto requiere hoy día es lo que no recibe ni está preparado para exigir en un medio ambiente formativo como el dominicano; a saber, capacidad de análisis y de razonamiento conducente al entendimiento de las cosas y de los procesos como son. Desprovisto de criterio o entendimiento propio, lo que se conoce como bombardeo mediático y de las redes sociales uniforman cada día más deseos y aspiraciones, apreciaciones, sentimientos, opiniones e ideas de sujetos tipificados y fácilmente hackeables en el país y desde el ciber espacio. Esa es la razón debido a la cual el progreso y la modernización terminan ofuscando al mismo tiempo el entendimiento y la libre decisión de cada uno de los que suman las grandes mayorías.

Esos agujeros negros sitúan la ESD ante una encrucijada. En poco tiempo, las universidades y demás IES ni siquiera serán capaces de vender la ilusión y muchos menos la esperanza de aproximar exitosamente al estudiantado al próximo mercado laboral en algo que no sean puestos de baja calificaciones que seguirán expulsando del suelo patrio a los mejores y más arriesgados exponentes del porvenir.

\section{El punto crítico: la utilidad de la ESD}

El mundo y la sociedad dominicana cambian a paso acelerado y por eso es menester contextualizar el impacto social de la ESD. No porque me cuestione, como tantos otros, si ha llegado el fin de la educación superior con el advenimiento de las nuevas tecnologías (Granados, 2019) y la robótica (Keane, 2019), —que no está por llegar-, sino porque me inquieta 
la permanencia vegetativa de valiosas instituciones de antaño expuestas a devenir irrelevantes - a no ser que cambien de rumbo-.

Para contextualizar e ilustrar aquel impacto, baste un ejemplo real y significativo. Me refiero a lo que acontece estos días en el país con el DR-Cafta (Dominican Republic-Central America Free Trade Agreement).

\section{a. Un ejemplo significativo}

Cierto, dicho acuerdo comercial fue firmado desde una posición de vulnerabilidad, lo que en su momento se denominó como docking. Así y todo, al igual que con los países centroamericanos pactantes, en la mesa coja de las negociaciones con la delegación dominicana se concedieron aproximadamente dos décadas para hacer más competitivos toda una estructura y serie de productos - principalmente agrícolas- en el naciente mercado regional.

¿Por qué traer a colación ese acontecimiento a propósito de la ESD? Dado que — al margen de cuestiones de política económica- lo acontecido es fiel reflejo del atavismo cultural que refuerza la distancia abismal que separa la academia dominicana de la economía. Cada sector, - el educativo en general y el universitario en particular, de una parte, y de la otra el productivo con sus extremos empresariales y laborales_-, permanece indiferente y ajeno al otro.

Esa indiferencia explica que llegáramos in fraganti a las respectivas fechas de desgravación de listas enteras de productos sin que mediara el indispensable esfuerzo de superación de todo un proceso de modernización productiva e industrial que - exagero para hacer valer el argumento- se resiste a competir en un mercado relativamente más libre y global. Como si el mercado local, formalmente abierto en el papel de los acuerdos comerciales, aún pudiera seguir siendo protegido por medio de arcanas modalidades de poder e influencias patrias, sobrevivientes todas de su hoy por hoy ilusorio e inexistente pasado.

En ese contexto crítico - aislamiento economía/ ESD — aventuro como posible explicación de ese fenómeno la siguiente hipótesis:

El desempeño de la ESD —a pesar de todo su legítimo empeño profesionalizante- está encaminado a llegar a ser superfluo e insignificante en términos de país, por dos realidades interdependientes y retroalimentadas entre sí:

1. Las IES dominicana dependen de y están determinadas por asuntos más administrativos $\mathrm{y}$ financieros que académicos $\mathrm{y}$, por ende, el efectivo desentendimiento y desarticulación entre sus tradicionales funciones de docencia, investigación y servicio comunitario. ${ }^{\mathrm{J}}$

2. El verificable desinterés de todo un sector empresarial que ni siquiera invierte en la mejoría de sus recursos humanos y en investigaciones tecnológicas llamadas a contribuir en la renovación de sus respectivos negocios en términos de competitividad y de calidad.

Dado el interés neurálgico al que apuntan, conceptualmente, el desenlace final y las dos realidades subyacentes a la ESD según la hipótesis expuesta, abro un largo paréntesis en la exposición, confiando que pueda, de algún modo, esclarecer asuntos de particular interés a especialistas en temas propios a la formación superior.

\section{b. Tradiciones subyacentes a la ESD}

Confirmar la veracidad de las dos realidades que avalan la hipótesis relativa al valor relevante o irrelevante de la ESD conlleva discernir la tradición occidental en la que se inscribe esa formación no ya desde tiempos de su inicio medieval ${ }^{\mathrm{K}} \mathrm{o}$ bien durante

J. Se trata de un problema de hecho, no conceptual. A nivel conceptual se reconoce - de acuerdo por ejemplo con el paradigma predominante de una reconocida universidad dominicana - "la investigación como una estrategia metodológica para el proceso de enseńanza-aprendizaje" (PUCMM, 2009). Sin embargo, en la práctica, la investigación está de facto divorciada del quehacer docente y no cuenta con respaldo porcentualmente significativo en los presupuestos institucionales. Por añadidura, se permanece disimulando la razón de ser de la educación superior pues, tal y como advierte la pregunta retórica de Pedro Henríquez Ureña, “¿en qué puede fundar una Universidad su reputación, si no en la sabiduría, libre de toda traba, de sus profesores?" (citada en Fiallo, 2019). En función de esa y de otras limitantes operativas del SESD, ver las propuestas de transformación de ese sistema resumidas de manera concisa por Leonardo Díaz, (2012).

K. En la sociedad europea, antes del siglo XII, la formación humanística y teológica se transmitía en los monasterios y, si acaso, en las escuelas catedralicias. Sin embargo, entrado el siglo XII surge una nueva realidad social: las ciudades, a las que la enseńanza da respuesta por medio de un nuevo tipo de asociación o gremio de profesores y estudiantes que pasa a ser reconocido como universidad. Las universidades pronto se institucionalizan como asociaciones de diversos saberes y escuelas, aunados todos por un mismo interés: la búsqueda de la verdad y de lo que en aquel entonces era concebido como la ciencia. 
la colonia española, sino al menos comenzando en una época más cercana al siglo xxi. Esa procedencia más reciente, aunque sea evocada a vuelo de pluma, es la siguiente:

Luego del tiranicidio de 1961 y la subsecuente revolución de abril del 65 , el modelo de educación superior napoleónico irrumpió en el país con evidente retraso respecto a lo sucedido en la Europa imperial; incluso, con el movimiento estudiantil de Córdoba, Argentina, el 21 de junio de 1918.

Ese modelo proveyó sentido social e histórico al acontecer universitario y a sus programas de estudio tanto en América Latina como en la República Dominicana, en particular. Pero en gran medida fue ese mismo modelo volcado en su impacto sociopolítico el que contribuyó al rezago hemisférico. ${ }^{\mathrm{L}}$ En vivo contraste con lo que acontecía localmente, a nivel internacional países punteros dedicaban su dinamismo académico a ralentizar el motor de las luchas sociales y a sustituirlo por la generación científica y tecnológica.

Allende los mares de las playas locales, fue así que se impuso a nivel mundial la revolución científico-tecnológica, con su tarea de transformar vertiginosamente el diario vivir de las poblaciones por medio de la destrucción creativa de un régimen económico que depende de la alianza entre la generación de nuevos saberes y su eficiente puesta en funcionamiento en el aparato productivo, bajo el legítimo manto de regímenes democráticos.

Ahora bien, lo significativo de ese maremágnum de acontecimientos es que, aunque el modelo napoleónico proporcionó e incluso podría seguir dotando de orientación a cierto mundo académico dominicano, no por ello supera la antinomia entre dos lados omnipresentes en los claustros universitarios y de educación superior en el país. Los lados desarticulados son la investigación y la docencia, imprescindibles para brindar servicio a la comunidad. La oposición de ambos extremos se debe, a mi mejor entender, a la organización universitaria heredera de la concepción de Immanuel Kant, tal y como la expuso el filósofo de Königsberg en su obra El Conflicto de las Facultades (1794).

L. Las escasas excepciones aparecen en contados centros universitarios de México, Brasil, Uruguay, Argentina, Chile, Costa Rica y Trinidad y Tobago.
Según Kant, la universidad se compone de facultades o espacios colegiados debidamente administrados. Siendo facultades docentes, la tarea encomendada se circunscribe a la formación en una u otra disciplina y ámbito profesional, resistiendo desde los recintos académicos la interferencia del poder gubernamental $\mathrm{u}$ otro cualquiera ${ }^{\mathrm{M}}$. Por vía de consecuencia, primero, se rompe con el modelo napoleónico dependiente del contexto social, así como con la investigación que procura la solución de problemas y la pesquisa de nuevos conocimientos y tecnologías, de modo que la educación superior se concentra y subordina a la enseñanza profesionalizante por facultades académicas.

En la línea de sucesión de la concepción de Kant, y más cercana a nosotros por su raigambre ibérica, José Ortega y Gasset en Misión de la Universidad (1930) advirtió que el énfasis en la investigación universitaria soslaya en la práctica lo que él promovía por antonomasia; es decir, la transmisión de la cultura y la enseñanza profesionalizante por las que él abogaba en su tierra natal.

Ambos modelos, el de Kant y el de Ortega y Gasset, —adjuntos al interés mercantil que a partir de los últimos tres o cuatro decenios del siglo $\mathrm{xx}$ se adueña de un país que cree poder institucionalizar-, conforman el sostén ideológico de todo el SESD. La formación profesional queda así entronizada como único pedestal y foco de atención de un sistema de enseñanza-aprendizaje que no hace otra cosa que no sea desalentar el estado de ánimo inconforme, útil e innovador del académico.

\section{c. La paradoja dominicana}

He ahílo que acontece en la República Dominicana. Su realidad educativa es paradójica. Si bien la vocación formativa y el espíritu esmerado y creativo del académico que es docente e investigador están desvalorizados en la sociedad dominicana y, como simple reflejo en su SESD, ambos son los primeros en ser reconocidos y aupados en las instituciones de formación superior de países donde prevalece una continua y revolucionaria construcción y destrucción de todo el orden axiológico e institucional de la civilización contemporánea.

M. Sea ese otro poder de índole empresarial, religioso u otro análogo. 
Por consiguiente, finalizo reasumiendo así aquel ejemplo del DR-Cafta y ese largo esbozo de los modelos ideales de formación superior: si la sociedad dominicana llegara a poder cambiar el interés y modelo operativo de sus centros de enseñanza superior, estaría en mejores condiciones de asumir corresponsablemente los desafíos que en el presente desatiende, y dejaría de seguir hipotecando a futuro tanto su capital humano como su aparato productivo.

En cualquier hipótesis que sea verificable, por el momento la ESD, su sistema y por ańadidura el mismo país que los propicia permanecen retenidos por el pasado. En el statu quo imperante, su modalidad educativa profesionalizante y mercantilizada no da muestras de poder enfrentar de manera corresponsable — ni siquiera - la superación de desafíos tan acuciantes en términos de sociedad como los experimentados por la situación que padece su aparato productivo. Cortar las amarras con esa realidad pasada es el desafío, por excelencia, del SESD y, en particular, de su educación, pues no solo la pobreza nos hace padecer y malvivir, también la miseria e inopia del espíritu. Como queda por ilustrar, tanto lastre es fruto de una usanza educativa rezagada desde su concepción original e inútil aún hoy día a la hora de dar un paso al frente y avanzar con valor hacia el porvenir.

\section{Retos}

Reconciliar y realzar las figuras del profesor y del estudiante - léase bien: del docente de carrera académica como eje central de cada institución de educación superior y de los alumnos que ya no pueden ni deben ser vistos como meros receptáculos huecos y vacíos que aquellos deben ir llenando hasta que queden listos, preparados para saltar a la siguiente etapa en el mercado laboral — ha de ser el principal desafío específico de todos.

Tomando prestado lo escrito por Margarita Heinsen (2019) para el nivel escolar, el académico cumple su rol original cuando aprovecha y utiliza todos los recursos disponibles para promover la curiosidad, realizar investigación, desarrollar la capacidad de solución de problemas, la creatividad, entre otras muchas habilidades de sus discípulos y de todos ellos como un grupo.

Pero, ¿cómo acercarse a ese ideal de manera que la práctica didáctica supere el ejercicio rutinario del magister dixit y de la estéril copia o repetición de contenidos e ideas?

Mitigar cualquier rastro de ineficiencia e ineptitud, conlleva — como zapata de nuevos edificios para la ESD - superar satisfactoriamente dos retos fundamentales:

10 Profesorado. Concebir e implementar un régimen de ESD centrado en y operando alrededor de un cuerpo docente provisto de las más altas calificaciones posibles, contratado a tiempo completo y promovido competitivamente en función de la evaluación de su desempeño y carrera académica. Como profesores, servirán de pedagogos y maestros del estudiante singular y de los estudiantes en general; y por ańadidura, aquellos que además sean investigadores, están convocados a promover e idealmente a generar nuevos conocimientos de valor a nivel nacional e internacional.

De manera complementaria a ese reto, surge otro en función del actual proceso de enseñanza-aprendizaje en el país. Según este proceso, la relación profesor-estudiante — si se quiere ser realista y optimistaconsiste en alcanzar y desarrollar el potencial inherente al entendimiento humano.

2o Enseńanza. Enseñar al estudiantado del SESD a entender y analizar de manera objetiva y lógica lo que estudian y en lo que se adiestran. Los estudiantes podrán así deducir, concluir y juzgar con propiedad lo que saben y hacen. Al mismo tiempo, darán un paso de superación por medio del cual, de ser sujetos meramente receptores, cheques en blanco, vasijas vacías, adquirirán la capacidad —intermedia- de comprender los fenómenos que estudian y con los que interactúan.

Al ser capaz de entender cosas, eventos y procesos, el estudiantado se encontrará en la cúspide de lo que es realista esperar, circunstancialmente, por medio del SESD en el país; es decir, dominar un estadio educativo intermedio entre una práctica habitual y una por ahora inalcanzable. Dejará atrás la situación de facto 
en la que se desenvuelve consuetudinaria o habitualmente la ESD contemporánea, que consiste en copiar, repetir y memorizar lo que hay que estudiar. Y, una vez capacitado para entender lo que es, quedará en la antesala de un tercer ámbito del conocimiento, por ahora irreal y utópico, que implica llegar a cuestionar, explicar y renovar lo que ya entiende.

La apuesta, por tanto, consiste en afrontar los dos retos a la vez. Esa es la única vía razonable para, por medio de un profesorado idóneo y suficiente, frenar la caída libre que como tendencia implica una enseñanza incapaz de liberarse del pasado, cautivada retóricamente y distanciada de apremiantes cuestiones del presente. Mismo que el estudiando en general no entiende y con cuyas realidades ni siquiera está adiestrado para interactuar $-\mathrm{y}$ mucho menos para interferir- en tanto que sujeto y actor heredero de un porvenir que ya toca las puertas de la sociedad dominicana.

\section{Conclusión}

Finalizo respondiendo dos preguntas esenciales - ¿para qué y por qué promover la ESD por medio de un modelo operativo más libre y académico que mercantil y kantiano o napoleónico? -

No para seguir haciendo más de lo mismo y así acerarnos cada día más a las puertas dantescas del averno, sino con el propósito de potenciar la capacidad de entendimiento de sus beneficiarios y, gracias a esta, empedrar el camino ideal del saber, hasta que un día, aún lejano, el sistema y las instituciones educativas en el país sean peritos en generar y compartir nuevos conocimientos de cara al futuro.

Y por eso mismo, en orden a impedir que la praxis actual haga que el ESD vuelva a quemarse como imberbe en una prueba final, llega la hora de afrontar con eficiencia y sentido de responsabilidad lo que debe ser nuestro principal propósito a inicios del siglo xxi:

Concebir, planificar e implementar, paulatinamente, un ambiente de enseńanza-aprendizaje aupado por una mayoría de pedagogos a tiempo completo y con las más altas cualidades posibles que, velando por la promoción de un estudiantado - hoy por hoy sumido en una caricatura según la cual a duras pena sabe leer y escribir, discernir el bien del mal o realizar simples operaciones matemáticas - que se supere en cada institución de formación superior hasta restablecerle valía, calidad, relevancia y porvenir a todo el SESD, a la gran mayoría de sus integrantes y a la sociedad dominicana como tal.

He ahí, — hasta prueba en contrario_- el principio fundamental de cuanto pretenda ser reconocido en la República Dominicana como tarea impostergable de la educación superior. Su cristalización yace más allá del sinsentido que impera en el presente. Este se reproduce desprovisto de profesores y de estudiantes de tiempo exclusivo que se promuevan interactuando entre sí; progresa carente de investigaciones, de laboratorios y de publicaciones; y añora al mismo tiempo, tanto los recursos financieros que está lejos de poseer, como el más elemental aprecio social y valoración cultural del conocimiento. Y todo eso, debido a que no cuenta con mayor experiencia de lo que falazmente publicita ser: una vivencia propiamente académica articulada - por medio de sus hallazgos científico-tecnológicos y sus escuelas de pensamiento- al sistema social dominicano y al global.

Por irreflexivas condiciones como las que se viven en el país día a día, muy pocos se preocupan por lo más elemental: “¿De dónde vienen los buenos empleos?” (Acemoglu, 2019), es decir, los que tienen un alto valor agregado como base fundamental para el desarrollo y bienestar en el seno de la cuarta revolución industrial.

No obstante, "la imprescindible expansión de la oferta educativa” dominicana, esos empleos con valor agregado ni siquiera se acercan o están relacionados con los que promueve anualmente y de manera utilitaria el saber profesionalizante vigente en el país. Bien por el contrario, en adición si acaso de restrictivas competencias, la oferta educativa dominicana no garantiza que la gente tenga "un propósito y un significado de vida” (Acemoglu, 2019; compare con Moreno Arellano 2019).

Ahora bien, llegados a esa afirmación conclusiva, que no se pretenda contraargumentar aduciendo falta de recursos para dar un brusco giro de timón a la ESD y procurarle nuevos y mejores horizontes. Claro que no sobran recursos humanos ni ninguno, pero hay suficientes como para tomar las decisiones justas 
y emprender una nueva aventura humana en esta nación tan requerida de ideales y de entendimiento. Para acometer la tarea pendiente se necesita, ante todo, poder de decisión institucional y, a modo de remembranza del artesano medieval y el aprendiz, convivencia metódica y sistemática de profesores y estudiantes.

Por consiguiente, es menester admitir, mientras le llega la hora a esa acometida, que de seguir entretenidos por el inmediatismo y la improvisación, y de espaldas a la tendencia hacia la mala calidad y peores niveles de competitividad del SESD, avanzaremos en vano produciendo profesionales con destrezas y conocimientos para un mundo que dejó de existir y para otro que ni siquiera somos capaces de entender y anticipar. Ese es el verdadero fruto salido de una realidad en la que de hecho se desconoce el valor de la educación y el impacto de las ideas.

\section{Referencias}

Abreu Van Grieken, C. (2018). Políticas docentes en la República Dominicana: avances y desafíos. Revista Ciencia \& Educación, 2(2), 13-28.

Acemoglu, D. (2019). Where do good jobs come from? En Project Syndicate, [Consultada el 26 de abril 2019]. Recuperado de: https:// www.project-syndicate.org/commentary/ automation-vs-job-creation-by-daron-acemoglu-2019-04

Bell, D. (1976). The coming of post-industrial society: A venture in social forecasting. New York, EE. UU.: Basic Books.

Chalmers, D. y Dennett, D. (24 de abril de 2016). Superintelligence impossible? On possible minds: philosophy and AI. [Entrada de un blog] Edge. Recuperado de https://ssociologos. com/2016/04/24/la-influencia-de-la-globalizacion-en-la-educacion/

Collado, M. (27 de mayo de 2019). A manera de epílogo: Ideas luminosas de Pedro Henríquez Ureña. Acento.com. Recuperado de https:// acento.com.do/2019/opinion/8685610-a-manera-de-epilogo-ideas-luminosas-de-pedro-henriquez-urena/
Corcino, P. (4 de abril de 2019). El MEESCyT exhibe bajas notas en la inversión en investigación. $E l$ Dinero, pp. 16-17.

Corporación Latinobarómetro. (2018) Informe 2018. Recuperado de http://www.latinobarometro. org/lat.jsp

Cubero Vázquez, K. y Villanueva Monge, L. (2014). La evaluación cualitativa en el proceso enseñanza - aprendizaje como principio fundamental de una formación más humanista. Revista Nuevo Humanismo, 2(1), 37-50. doi: http://dx.doi. org/10.15359/rnh.2-1.3

Descartes, R. (2010). Discurso del método. [Traducción al español de Manuel García Morente]. Madrid, España: Colección Austral Espasa-Calpe.

Díaz, L. (8 de agosto de 2012). Propuestas para la transformación del sistema dominicano de educación superior. Acento.com. Recuperado de https:// acento.com.do/2012/opinion/205475-propuestas-para-la-transformacion-del-sistema-dominicano-de-educacion-superior/

Diz López, M., Valcarce Fernández, M. y Castro González, M. (2018). Valoración por el estudiantado de la estrategia de aprendizaje mediante proyectos de investigación frente a las clases expositivas con casos prácticos. Estudio de caso. Cuaderno de Pedagogía Universitaria, 15(30), 22-36.

Drucker, P. (1993). Post-capitalist society. Londres, Inglaterra: Routledge.

Educa. (2019). Los jóvenes dominicanos, esos desconocidos. "Estado de situación y políticas en materia de formación y empleo juvenil en la República Dominicana”. Recuperadodehttp://www.educa. org.do/los-jovenes-dominicanos-esos-desconocidos-estado-de-situacion-y-politicas-en-materia-de-formacion-y-empleo-juvenil-en-la-republica-dominicanal

García, C. (24 de abril de 2016). La influencia de la globalización en la educación. [Entrada de blog] Ssociólogos. Recuperado de https://ssociologos. com/2016/04/24/la-influencia-de-la-globalizacion-en-la-educacion/

Giroux, H. (2018). Pedagogía critica para tiempos difíciles. Madrid, España: Editorial Mapas Colectivos, Colección Microbooks. 
Giroux, H. (2005). Estudios culturales, pedagogía crítica y democracia radical. Madrid, España: Editorial Popular. Colección Proa.

Giroux, H. (2003). Pedagogía y política de la esperanza: teoría, cultura y enseñanza: una antología crítica. Buenos Aires, Argentina: Amorrortu.

Granados, O. (8 de febrero de 2019). ¿El fin de las universidades? El País. Recuperado de https:// elpais.com/internacional/2019/02/08/mexico/ 1549581066_521979.html

Harari, Y. (2018). 21 lecciones para el siglo XXI. Barcelona, España: Debate.

Heinsen, M. (12 de marzo de 2019). Quedarse sin recreo no es una opción. Acentro.com. Recuperado de https://acento.com.do/2019/ opinion/8659644-quedarse-sin-recreo-nouna-opcion/

Keane, J. (1 de abril 2019). La nueva era de la revolución de las máquinas. Letras Libres, (244). Recuperado dehttps://www.letraslibres.com/espana-mexico/ revista/la-nueva-era-la-revolucion-las-maquinas

Kant, I. (1794). ElConflicto de las Facultades, [Traducción al español de R. Rodríguez Aramayo, 2003]. Madrid, España: Alianza Editorial.

Moreno Arellano, C. (10 de mayo de 2019). Educación, empleo y desarrollo: No hay atajos. Milenio. Recuperadodehttps:/www.milenio.com/opinion/ carlos-ivan-moreno-arellano/dialogo-publico/ educacion-empleo-y-desarrollo-no-hay-atajos

Murray, G. (2005). El colegio y la escuela: Antropología de la educación en la República Dominicana. Santo Domingo, República Dominicana: FondoMicro, Amigo del Hogar.

Observatorio de Políticas Sociales y Desarrollo de la Vicepresidencia de la República Dominicana. (2019). Educación superior dominicana: expansión, desarrollo y perspectivas futuras 3(1), $12 \mathrm{pp}$.
Oppenheimer, A. (2018). ¡Sálvese quien pueda! El futuro del trabajo en la era de la Automatización. Madrid, España: Debate.

Ortega y Gasset, J. (1939). La rebelión de las masas. Madrid, España: Espasa-Calpe, Colección Austral.

PISA Worldwide Ranking - average score of math, science and reading. Recuperado de http:// factsmaps.com/pisa-worldwide-ranking-average-score-of-math-science-reading/

Pontificia Católica Universidad Madre y Maestra, (PUCMM). (2009). Modelo Educativo. Santiago de los Caballeros, República Dominicana: PUCMM.

Tavares, J. (23 de mayo de 2019). ¡Que viva la mediocridad! Diario Libre. Recuperado de https:// www.diariolibre.com/opinion/en-directo/ que-viva-la-mediocridad-EI12837773

Toffler, A. (1984). El schock del futuro. Barcelona, España: Plaza y Janés.

Torres Menárguez, A. (14 de mayo de 2019). La crisis de la escuela es la crisis de la democracia. El País. Recuperado de https://elpais. com/sociedad/2019/05/09/actualidad/ 1557407024_184967.html

Vargas, E. (13 de mayo de 2019). Educación basada en competencias: ¿hacia el éxito o fracaso? Acento.com. Recuperado de https://acento.com. do/2019/opinion/8681268-educacion-basada-en-competencias-hacia-el-exito-o-fracaso/

Weber, M. (1904). La ética protestante y el espiritu del capitalismo. [Traducción al español de Joaquín Abellán, 2012]. Madrid, España: Alianza Editorial. 